\title{
Generalized Entropy of Order Statistics
}

\author{
Richa Thapliyal, H. C. Taneja \\ Department of Applied Mathematics, Delhi Technological University, Delhi, India \\ Email: richa31aug@gmail.com; hctaneja@rediffmail.com
}

Received September 4, 2012; revised October 12, 2012; accepted October 20, 2012

\begin{abstract}
In this communication, we consider and study a generalized two parameters entropy of order statistics and derive bounds for it. The generalized residual entropy using order statistics has also been discussed.
\end{abstract}

Keywords: Entropy; Order Statistics; Probability Integral Transformation; Residual Entropy; Generalized Information

\section{Introduction}

Suppose $X_{1}, X_{2}, \cdots, X_{n}$ are $n$ independent and identically distributed observations from a distribution $F_{X}$, where $F_{X}$ is differentiable with a density $f_{X}$ which is positive in an interval and zero elsewhere. The order statistics of the sample is defined by the arrangement of $X_{1}, X_{2}, \cdots, X_{n}$ from the smallest to largest denoted as $X_{1: n}, X_{2: n}, \cdots, X_{n: n}$. Then the p.d.f. of the $i^{\text {th }}$ order statistics $X_{i: n}$, is given by

$$
\begin{aligned}
f_{i: n}(y) & =\frac{1}{B(i, n-i+1)}\left[F_{X}(y)\right]^{i-1} \\
& \cdot\left[1-F_{X}(y)\right]^{n-i} f_{X}(y),
\end{aligned}
$$

for details refer to [1].

Order statistics has been studied by statisticians for some time and has been applied to problems of statistical estimation [2], reliability analysis, image coding [3] etc. Some information theoretic aspects of order statistics have been discussed in the literature. Wong and Chen [4] showed that the difference between average entropy of order statistics and the entropy of a data distribution is a constant. Park [5] showed some recurrence relations for entropy of order statistics. Information properties of order statistics based on Shannon entropy [6] and Kullback-Leibler [7] measure using probability integral transformation have been studied by Ebrahimi et al. [8]. Arghami and Abbasnejad [9] studied Renyi entropy properties based on order statistics. The Renyi [10] entropy is a single parameter entropy. We consider a generalized two parameter, the Verma entropy [11], and study it in context with order statistics. Verma entropy plays a vital role as a measure of complexity and uncertainty in different areas such as physics, electronics and engineering to describe many chaotic systems. Considering the importance of this entropy measure, it will be worthwhile to study it in case of order statistics. The rest of the article is organized as follows:

In Section 2, we express generalized entropy of $i^{\text {th }}$ order statistics in terms of generalized entropy of $i^{\text {th }}$ order statistics of uniform distribution and study some of its properties. Section 3 provides bounds for entropy of order statistics. In Section 4, we derive an expression for residual generalized entropy of order statistics using residual generalized entropy for uniform distribution.

\section{Generalized Entropy of Order Statistics}

Let $X$ be a random variable having an absolutely continuous cdf $F(x)$ and pdf $f(x)$, then Verma [11] entropy of the random variable $X$ with parameters $\alpha, \beta$ is defined as:

$$
\begin{aligned}
& H_{\alpha}^{\beta}(X)=-\frac{1}{\alpha-\beta} \log \int_{0}^{\infty} f^{\alpha+\beta-1}(x) \mathrm{d} x, \forall \beta \geq 1, \\
& \alpha \neq \beta, \beta-1<\alpha<\beta,
\end{aligned}
$$

where

$$
\lim _{\beta=1} H_{\alpha}^{\beta}(X)=H_{\alpha}(X)=-\frac{1}{\alpha-1} \log \int_{0}^{\infty} f^{\alpha}(x) \mathrm{d} x,
$$

is the Renyi entropy, and

$$
\lim _{\beta=1, \alpha \rightarrow 1} H_{\alpha}^{\beta}(X)=-\int_{0}^{\infty} f(x) \log f(x) \mathrm{d} x,
$$

is the Shannon entropy .

We use the probability integral transformation of the random variable $U=F(X)$ where the distribution of $U$ is the standard uniform distribution. If $V_{1}, V_{2}, \cdots, V_{n}$ are the order statistics of a random sample $U_{1}, U_{2}, \cdots, U_{n}$ from uniform distribution, then it is easy to see using (1) that $V_{i}, i=1,2, \cdots, n$ has beta distribution with parameters $i$ and $(n-i+1)$. Using probability integral transformation, entropy (2) of the random variable $X$ can be 
represented as

$$
H_{\alpha}^{\beta}(X)=-\frac{1}{\alpha-\beta} \log \int_{0}^{1} f^{\alpha+\beta-2}\left(F^{-1}(u)\right) \mathrm{d} u .
$$

Next, we prove the following result:

Theorem 2.1 The generalized entropy of $X_{i: n}$ can be expressed as

$$
H_{\alpha}^{\beta}\left(X_{i: n}\right)=H_{\alpha}^{\beta}\left(V_{i}\right)-\frac{1}{\alpha-\beta} \log E_{g_{i}}\left[f^{\alpha+\beta-2}\left(F^{-1}\left(Z_{i}\right)\right)\right],
$$

where $H_{\alpha}^{\beta}\left(V_{i}\right)$ denotes the entropy of the beta distribution with parameters $i$ and $(n-i+1), E_{g_{i}}(X)$ denotes expectation of $X$ over $g_{i}$ and $Z_{i} \sim g_{i}$ is the beta density with parameters $(\alpha+\beta-1)(i-1)+1$ and $(\alpha+\beta-1)(n-i)+1$

Proof: Since $V_{i}=F\left(X_{i: n}\right), i=1,2, \cdots, n$ which implies $X_{i: n}=F^{-1}\left(V_{i}\right)$. Thus, from (3) we have

$$
\begin{aligned}
H_{\alpha}^{\beta}\left(X_{i: n}\right)= & -\frac{1}{\alpha-\beta} \log \left[\frac{\Gamma(n+1)}{\Gamma(i) \Gamma(n-i+1)}\right]^{\alpha+\beta-1}-\frac{1}{\alpha-\beta} \log \int_{0}^{1} v_{i}^{(\alpha+\beta-1)(i-1)}\left(1-v_{i}\right)^{(\alpha+\beta-1)(n-i)} f^{(\alpha+\beta-2)}\left(F^{-1}\left(v_{i}\right)\right) \mathrm{d} v_{i} \\
= & -\frac{(\alpha+\beta-1)}{\alpha-\beta} \log \left[\frac{\Gamma(n+1)}{\Gamma(i) \Gamma(n-i+1)}\right]+\frac{1}{\alpha-\beta} \log \left[\frac{\Gamma((\alpha+\beta-1)(n-1)+2)}{\Gamma((\alpha+\beta-1)(i-1)+1) \Gamma((\alpha+\beta-1)(n-i)+1)}\right] \\
& -\frac{1}{\alpha-\beta} \log \int_{0}^{1}\left[\frac{\Gamma(\alpha+\beta-1)(n-1)+2}{\Gamma((\alpha+\beta-1)(i-1)+1) \Gamma((\alpha+\beta-1)(n-i)+1)}\right] \\
& \times z_{i}^{(\alpha+\beta-1)(i-1)}\left(1-z_{i}\right)^{(\alpha+\beta-1)(n-i)} f^{(\alpha+\beta-1)}\left(F^{-1}\left(z_{i}\right)\right) \mathrm{d} z_{i} .
\end{aligned}
$$

It is easy to see that the entropy (2) for the beta distribution with parameters $i$ and $(n-i+1)$ (that is, the $i^{\text {th }}$ order statistics of uniform distribution) is given by

$$
H_{\alpha}^{\beta}\left(V_{i}\right)=\left(\frac{\alpha+\beta-1}{\alpha-\beta}\right) \log B(i, n-i+1)-\frac{1}{\alpha-\beta} \log B((\alpha+\beta-1)(i-1)+1,(\alpha+\beta-1)(n-i)+1) .
$$

Using (6) in (5), the desired result (4) follows.

In particular, by taking $\beta=1$ and $\alpha \rightarrow 1$, (4) reduces to

$$
H\left(X_{i: n}\right)=H\left(V_{i}\right)-E_{g_{i}}\left[\log f\left(F^{-1}\left(Z_{i}\right)\right)\right],
$$

a result derived by Ebrahimi et al. [8].

Remark: In reliability engineering $(n-k+1)$-outof- $n$ systems are very important kind of structures. A $(n-k+1)$-out-of- $n$ system functions iff atleast $(n-k+1)$ components out of $n$ components function. If $X_{1}, X_{2}, \cdots, X_{n}$ denote the independent lifetimes of the components of such system, then the lifetime of the system is equal to the order statistic $X_{k: n}$. The special case of $k=1$ and $n$, that is for sample minima and maxima correspond to series and parallel systems respectively. In the following example, we calculate entropy (4) for sample maxima and minima for an exponential distribution.

Example 2.1 Let $X$ be a random variable having the exponential distribution with pdf

$$
f(x)=\theta \mathrm{e}^{-\theta x}, \theta>0, x \geq 0 .
$$

Here, $F^{-1}(z)=-\theta^{-1} \log (1-z)$ and the expectation term is given by

$$
E_{g_{i}}\left[f^{\alpha+\beta-2}\left(F^{-1}\left(Z_{i}\right)\right)\right]=E_{g_{i}}\left[\theta^{\alpha+\beta-2}\left(1-Z_{i}\right)^{\alpha+\beta-2}\right]=\theta^{\alpha+\beta-2} \frac{B((\alpha+\beta-1)(i-1)+1,(\alpha+\beta-1)(n-i+1))}{B((\alpha+\beta-1)(i-1)+1,(\alpha+\beta-1)(n-i)+1)} .
$$

For $i=1$, from (6), we have

$$
\begin{aligned}
H_{\alpha}^{\beta}\left(V_{1}\right) & =-\frac{1}{\alpha-\beta} \\
& \cdot[(\alpha+\beta-1) \log n-\log ((\alpha+\beta-1)(n-1)+1)] .
\end{aligned}
$$

Hence, using (4)

$$
H_{\alpha}^{\beta}\left(X_{1: n}\right)=-\left(\frac{\alpha+\beta-2}{\alpha-\beta}\right) \log n \theta+\frac{\log (\alpha+\beta-1)}{(\alpha-\beta)},
$$

which confirms that the sample minimum has an exponential distribution with parameter $n \theta$, since 


$$
H_{\alpha}^{\beta}(X)=-\left(\frac{\alpha+\beta-2}{\alpha-\beta}\right) \log \theta+\frac{\log (\alpha+\beta-1)}{\alpha-\beta},
$$

where $X$ is an exponential variate with parameter $\theta$.

Also

$$
H_{\alpha}^{\beta}\left(X_{1: n}\right)-H_{\alpha}^{\beta}(X)=\left(\frac{\alpha+\beta-2}{\beta-\alpha}\right) \log n .
$$

Hence, the difference between the generalized entropy of first order statistics i.e. the sample minimum and the generalized entropy of parent distribution is independent of parameter $\theta$, but it depends upon sample size $n$. Similarly, for sample maximum, we have

$$
\begin{aligned}
& H_{\alpha}^{\beta}\left(X_{n: n}\right)=-\left(\frac{\alpha+\beta-1}{\alpha-\beta}\right) \log n-\left(\frac{\alpha+\beta-2}{\alpha-\beta}\right) \log \theta \\
& -\frac{1}{(\alpha-\beta)} \log B((\alpha+\beta-1),(\alpha+\beta-1)(n-1)+1) .
\end{aligned}
$$

It can be seen easily that the difference between $H_{\alpha}^{\beta}\left(X_{n: n}\right)$ and $H_{\alpha}^{\beta}(X)$ is

$$
\begin{aligned}
& -\left(\frac{\alpha+\beta-1}{\alpha-\beta}\right) \log n \\
& -\frac{1}{(\alpha-\beta)} \log B((\alpha+\beta-1),(\alpha+\beta-1)(n-1)+1) \\
& -\frac{\log (\alpha+\beta-1)}{\alpha-\beta}
\end{aligned}
$$

$$
\begin{aligned}
g_{i}(z) & \leq B_{i}=g_{i}\left(m_{i}\right) \\
& =\frac{1}{B((\alpha+\beta-1)(i-1)+1,(\alpha+\beta-1)(n-i)+1)} m_{i}^{(\alpha+\beta-1)(i-1)+1}\left(1-m_{i}\right)^{(\alpha+\beta-1)(n-i)+1} .
\end{aligned}
$$

For $\beta>1, \beta-1<\alpha<\beta$, from (4)

$$
\begin{aligned}
H_{\alpha}^{\beta}\left(X_{i: n}\right)-H_{\alpha}^{\beta}\left(V_{i}\right) & =-\frac{1}{\alpha-\beta} \log \int g_{i}(z) f^{\alpha+\beta-2}\left(F^{-1}(z)\right) \mathrm{d} z \leq-\frac{1}{\alpha-\beta} \log B_{i} \int f^{\alpha+\beta-2}\left(F^{-1}(z)\right) \mathrm{d} z \\
& =-\frac{1}{\alpha-\beta} \log B_{i}-\frac{1}{\alpha-\beta} \log \int f^{\alpha+\beta-2}\left(f^{-1}(z)\right) \mathrm{d} z \\
& =-\frac{1}{\alpha-\beta} \log B_{i}-\frac{1}{\alpha-\beta} \log \int f^{\alpha+\beta-1}(x) \mathrm{d} x=-\frac{1}{\alpha-\beta} \log B_{i}+H_{\alpha}^{\beta}(X) .
\end{aligned}
$$

which gives (7).

From (4) we can write

$$
H_{\alpha}^{\beta}\left(X_{i: n}\right) \geq H_{\alpha}^{\beta}\left(V_{i}\right)-\left(\frac{1}{\alpha-\beta}\right) \log \int g_{i}(v) M^{\alpha+\beta-2} \mathrm{~d} v=H_{\alpha}^{\beta}\left(V_{i}\right)-\left(\frac{\alpha+\beta-2}{\alpha-\beta}\right) \log M .
$$

Example 3.1 For the uniform distribution over the interval $[a, b]$ we have

$$
H_{\alpha}^{\beta}(X)=\left(\frac{\alpha+\beta-2}{\alpha-\beta}\right) \log (b-a),
$$

and from (6),

$$
\begin{aligned}
& H_{\alpha}^{\beta}\left(V_{1}\right)=H_{\alpha}^{\beta}\left(V_{n}\right)=-\left(\frac{1}{\alpha-\beta}\right) \\
& \cdot[(\alpha+\beta-1) \log n-\log ((\alpha+\beta-1)(n-1)+1)] .
\end{aligned}
$$


and

$$
C_{1}=C_{n}=-\left(\frac{\alpha+\beta-1}{\alpha-\beta}\right) \log n .
$$

Hence, using (7) we get

$$
H_{\alpha}^{\beta}\left(X_{1: n}\right) \leq-\left(\frac{\alpha+\beta-1}{\alpha-\beta}\right) \log n+\left(\frac{\alpha+\beta-2}{\alpha-\beta}\right) \log (b-a) .
$$

Further, for uniform distribution over the interval $[a, b], \quad M=\frac{1}{b-a}$. Using (8) we get

$$
H_{\alpha}^{\beta}\left(X_{1: n}\right) \geq-\frac{1}{\alpha-\beta}[(\alpha+\beta-1) \log n-\log ((\alpha+\beta-1)(n-1)+1)]+\left(\frac{\alpha+\beta-2}{\alpha-\beta}\right) \log (b-a) .
$$

Thus, for uniform distribution, we have

$$
\begin{aligned}
& -\frac{1}{\alpha-\beta}[(\alpha+\beta-1) \log n-\log ((\alpha+\beta-1)(n-1)+1)]+\left(\frac{\alpha+\beta-2}{\alpha-\beta}\right) \log (b-a) \\
\leq & H_{\alpha}^{\beta}\left(X_{1: n}\right) \leq-\left(\frac{\alpha+\beta-1}{\alpha-\beta}\right) \log n+\left(\frac{\alpha+\beta-2}{\alpha-\beta}\right) \log (b-a) .
\end{aligned}
$$

We can check that the bounds for $H_{\alpha}^{\beta}\left(X_{n: n}\right)$ are same as that of $H_{\alpha}^{\beta}\left(X_{1: n}\right)$.

Example 3.2 For the exponential distribution with parameter $\theta$, we have $M=\theta$ and

$$
\begin{gathered}
H_{\alpha}^{\beta}(X)=-\left(\frac{\alpha+\beta-1}{\alpha-\beta}\right) \log \theta+\frac{\log ((\alpha+\beta-1) \theta)}{\alpha-\beta} . \quad \text { Using Theorem 3.1 } \\
\quad-\left(\frac{1}{\alpha-\beta}\right)[(\alpha+\beta-1) \log n-\log ((\alpha+\beta-1)(n-1)+1)]-\left(\frac{\alpha+\beta-2}{\alpha-\beta}\right) \log \theta \\
\leq H_{\alpha}^{\beta}\left(X_{1: n}\right) \leq-\left(\frac{\alpha+\beta-1}{\alpha-\beta}\right) \log n-\left(\frac{\alpha+\beta-2}{\alpha-\beta}\right) \log \theta+\frac{\log (\alpha+\beta-1)}{\alpha-\beta} .
\end{gathered}
$$

Here we observe that the difference between upper bound and $H_{\alpha}^{\beta}\left(X_{1: n}\right)$ is $\left(\frac{1}{\beta-\alpha}\right) \log n$, which is an increasing function of $n$. Thus, for the exponential distribution upper bound is not useful when sample size is large.

\section{The Generalized Residual Entropy of Order Statistics}

In reliability theory and survival analysis, $X$ usually denotes a duration such as the lifetime. The residual lifetime of the system when it is still operating at time $t$, given by $X_{t}=(X-t \mid X>t)$ has the probability density $f(x ; t)=\frac{f(x)}{\bar{F}(t)}, x \geq t>0$, where $\bar{F}(t)=1-F(t)>0$.

Ebrahimi [12] proposed the entropy of the residual lifetime $X_{t}$ as

$$
H(X ; t)=-\int_{t}^{\infty} \frac{f(x)}{\bar{F}(t)} \log \frac{f(x)}{\bar{F}(t)} \mathrm{d} x, t>0 .
$$

Thus, as calculated in Example 2.1

$$
\begin{aligned}
H_{\alpha}^{\beta}\left(X_{1: n}\right) & =-\left(\frac{\alpha+\beta-2}{\alpha-\beta}\right) \log n \theta \\
& +\frac{\log (\alpha+\beta-1)}{\alpha-\beta} .
\end{aligned}
$$

Obviously, when $t=0$, it reduces to Shannon entropy.

The generalized residual entropy of the type $(\alpha, \beta)$ is defined as

$$
H_{\alpha}^{\beta}(X ; t)=\frac{1}{\beta-\alpha} \log \int_{t}^{\infty} \frac{f^{\alpha+\beta-1}(x)}{\bar{F}^{\alpha+\beta-1}(t)} \mathrm{d} x,
$$

where $\beta-1<\alpha<\beta, \beta \geq 1$. When $t=0$, it reduces to (2).

We note that the density function and survival function of $X_{i: n}$ (refer to [13]), denoted by $f_{i: n}(x)$ and $\bar{F}_{i: n}(x), i=1,2,3, \cdots, n$, respectively are

$$
f_{i: n}(x)=\frac{1}{B(i, n-i+1)}[f(x)]^{i-1}[1-F(x)]^{n-i} f(x),
$$

where

$$
B(a, b)=\int_{0}^{1} x^{a-1}(1-x)^{b-1} \mathrm{~d} x, a>0, b>0,
$$

and

$$
\bar{F}_{i: n}(x)=\frac{\bar{B}_{F(x)}(i, n-i+1)}{B(i, n-i+1)},
$$


where

$$
\bar{B}_{x}(a, b)=\int_{x}^{1} u^{a-1}(1-u)^{b-1} \mathrm{~d} u, 0<x<1 .
$$

$B(a, b)$ and $\bar{B}_{x}(a, b)$ are known as the beta and incomplete beta functions respectively. In the next lemma, we derive an expression for $H_{\alpha}^{\beta}\left(V_{i} ; t\right)$ for the dynamic version of $H_{\alpha}^{\beta}\left(V_{i}\right)$ as given by (6).

Lemma 4.1 Let $V_{i}$ be the $i^{\text {th }}$ order statistics based on a random sample of size $n$ from uniform distribution on $(0,1)$. Then

$$
H_{\alpha}^{\beta}\left(V_{i} ; t\right)=\frac{1}{\beta-\alpha} \log \bar{B}_{t}((\alpha+\beta-1)(i-1)+1,(\alpha+\beta-1)(n-i)+1)-\left(\frac{\alpha+\beta-1}{\beta-\alpha}\right) \log \bar{B}_{t}(i, n-i+1) .
$$

Proof: For uniform distribution using (10), we have

$$
H_{\alpha}^{\beta}\left(V_{i} ; t\right)=\frac{1}{\beta-\alpha} \log \int_{t}^{\infty} \frac{f_{i: n}^{\alpha+\beta-1}(x)}{\bar{F}^{\alpha+\beta-1}(t)} \mathrm{d} x
$$

Putting values from (11) and (13) in (16), we get the desired result (15).

If we put $t=0$ in (15), we get (6).
Using this, in the following theorem, we will show that the residual entropy of order statistics $X_{i: n}$ can be represented in terms of residual entropy of uniform distribution.

Theorem 4.1 Let $F$ be an absolutely continuous distribution function with density $f$. Then, generalized residual entropy of the $i^{\text {th }}$ order statistics can be represented as

$$
H_{\alpha}^{\beta}\left(X_{i: n} ; t\right)=H_{\alpha}^{\beta}\left(V_{i} ; F(t)\right)+\frac{1}{\beta-\alpha} \log E\left[f^{\alpha+\beta-2}\left(F^{-1}\left(Y_{i}\right)\right)\right]
$$

where

$$
Y_{i} \sim \bar{B}_{F(t)}((\alpha+\beta-1)(i-1)+1,(\alpha+\beta-1)(n-i)+1) .
$$

Proof: Using the probability integral transformation

$$
V_{i}=F\left(X_{i: n}\right), i=1,2,3, \cdots, n
$$

and above lemma, the result follows.

Take $t=0$ in (17), it reduces to (4).

Example 4.1 Suppose that $X$ is exponentially distributed random variable with mean $\frac{1}{\theta}$. Then,

$$
f\left(F^{-1}(y)\right)=\theta(1-y)
$$

and we have

$$
\begin{aligned}
& E\left[f^{\alpha+\beta-2}\left(F^{-1}\left(Y_{1}\right)\right)\right] \\
& =\frac{\theta^{\alpha+\beta-2} \mathrm{e}^{-\theta t(\alpha+\beta-2)}}{n(\alpha+\beta-1)[(\alpha+\beta-1)(n-1)+1]} .
\end{aligned}
$$

For $i=1$, Theorem 4.1 gives

$$
\begin{aligned}
H_{\alpha}^{\beta}\left(X_{1: n} ; t\right) & =-\frac{1}{\beta-\alpha} \log (\alpha+\beta-1) \\
& +\left(\frac{\alpha+\beta-2}{\beta-\alpha}\right) \log n \theta .
\end{aligned}
$$

Also

$H_{\alpha}^{\beta}(X ; t)=\left(\frac{\alpha+\beta-2}{\beta-\alpha}\right) \log \theta-\frac{1}{\beta-\alpha} \log (\alpha+\beta-1)$.

Hence

$$
H_{\alpha}^{\beta}\left(X_{1: n} ; t\right)-H_{\alpha}^{\beta}(X ; t)=-\left(\frac{\alpha+\beta-2}{\beta-\alpha}\right) \log n .
$$

So, in the exponential case the difference between generalized residual entropy of the lifetime of a series system and residual generalized entropy of the lifetime of each component is independent of time.

\section{Conclusion}

The two parameters generalized entropy plays a vital role as a measure of complexity and uncertainty in different areas such as physics, electronics and engineering to describe many chaotic systems. Using probability integral transformation we have studied the generalized and generalized residual entropies based on order statistics. We have explored some properties of these entropies for exponential distribution.

\section{Acknowledgements}

The first author is thankful to the Center for Scientific and Industrial Research, India, to provide financial assistance for this work.

\section{REFERENCES}

[1] B. C. Arnold, N. Balakrishnan and H. N. Nagaraja, “A First Course in Order Statistics,” John Wiley and Sons, New York, 1992.

[2] E. H. Llyod, "Least-Squares Estimation of Location and Scale Parameters Using Order Statistics,” Biometrika, Vol. 39, No. 1-2, 1952, pp. 88-95. 
[3] E. Ataman, V. K. Aatre and K. M. Wong, "Some Statistical Properties of Median Filters," IEEE Transactions on Acoustics, Speech and Signal Processing, Vol. ASSP-29, No. 5, 1981, pp. 1073-1075.

[4] K. M. Wong and S. Chen, "The Entropy of Ordered Sequences and Order Statistics," IEEE Transactions on Information Theory, Vol. 36, No. 2, 1990, pp. 276-284. doi:10.1109/18.52473

[5] S. Park, "The Entropy of Consecutive Order Statistics," IEEE Transactions on Information Theory, Vol. 41, No. 6, 1995, pp. 2003-2007. doi:10.1109/18.476325

[6] C. E. Shannon, "A Mathematical Theory of Communication,” Bell System Technical Journal, Vol. 27, 1948, pp. 379-423 and 623-656.

[7] S. Kullback, "Information Theory and Statistics," Wiley, New York, 1959.

[8] N. Ebrahimi, E. S. Soofi and H. Zahedi, "Information Properties of Order Statistics and Spacings," IEEE Tran- sactions on Information Theory, Vol. 50, No. 1, 2004, pp. 177-183. doi:10.1109/TIT.2003.821973

[9] N. R. Arghami and M. Abbasnejad, "Renyi Entropy Properties of Order Statistics," Communications in Statistics, Vol. 40, No. 1, 2011, pp. 40-52. doi:10.1080/03610920903353683

[10] A. Renyi, "On Measures of Entropy and Information," Proceedings of the Fourth Berkeley Symposium on Mathematical Statistics and Probability, Berkley, 20 June-30 July 1961, pp. 547-561.

[11] R. S. Verma, "Generalization of Renyi's Entropy of Order $\alpha$,” Journal of Mathematical Sciences, Vol. 1, 1966, pp. 34-48.

[12] N. Ebrahimi, "How to Measure Uncertainty in the Residual Lifetime Distributions,” Sankhya A, Vol. 58, 1996, pp. 48-57.

[13] H. A. David and H. N. Nagaraja, "Order Statistics," Wiley, New York, 2003. doi:10.1002/0471722162 